\title{
High expression of HSP90 is associated with poor prognosis in patients with colorectal cancer
}

\author{
Shuming Zhang ${ }^{1}$, Shichao Guo ${ }^{1}$, Zhangfu $\mathbf{L i}^{1}{ }^{1}$, Dan Li ${ }^{\text {Corresp., }}{ }^{1}$, Qimin Zhan ${ }^{\text {Corresp. } 1,2}$ \\ ${ }^{1}$ State Key Laboratory of Molecular Oncology, National Cancer Center/National Clinical Research Center for Cancer/Cancer Hospital, Chinese Academy of \\ Medical Sciences and Peking Union Medical College, Beijing, China \\ ${ }^{2}$ Laboratory of Molecular Oncology, Key laboratory of Carcinogenesis and Translational Research (Ministry of Education/Beijing), Laboratory of Molecular \\ Oncology, Peking University Cancer Hospital \& Institute, Beijing, China \\ Corresponding Authors: Dan Li, Qimin Zhan \\ Email address: eileenld@gmail.com, zhanqimin@bjmu.edu.cn
}

Background: Heat shock protein 90 (HSP90) is a highly conserved chaperone with an approximate molecular weight of $90-\mathrm{kDa}$. It plays a critical role in maintaining stability and homeostasis of oncoproteins, helping cancer cells living in the unsuitable environmental conditions. The current study aims to inquire the difference of HSP90 expression in tumor tissues and normal tissues, analyze the correlation between HSP90 expression and the prognoses of patients with colorectal cancer (CRC), and investigate its role in CRC preliminarily. [b]

Methods: Online analysis of HSP90 mRNA levels in different cancers was firstly done in GEPIA. Then HSP90 expression was determined by immunohistochemistry between 99 colorectal cancer (CRC) tissues and 81 normal tissues. Chi-square test or Fisher's exact test was used to analyze the relationship between HSP90 and histopathologic characteristics. Kaplan-Meier analysis and Cox's proportional hazards model were also done for further analysis of the prognostic values of HSP90. Pearson's correlation coefficients between HSP90 expression values and other mRNA expression values were calculated based on TCGA dataset and bioinformatic analysis was done about these screened genes. [b]

Results: CRC tissues showed significantly higher expression of HSP90 than normal tissues ( 55.6 \% vs. $3.7 \%, \mathrm{P}<0.0001$ ). Kaplan-Meier curves showed high HSP90 expression was associated with poor prognosis $(\mathrm{P}=0.039)$ in $\mathrm{CRC}$ patients, and multivariate Cox proportional hazards regression model analysis also indicated that HSP90 expression $(\mathrm{HR}=1.93$ 0, 95\% Cl: $1.113-3.349, \mathrm{P}=0.019$ ) linked to poor prognosis. Moreover, 85 genes were correlated with HSP90, which were involved in metabolic process and enriched in pathways of Proteasome and Base excision repair. [b]

Conclusions : Our results suggested that HSP90 expression is inversely associated with survival outcomes and could be an independent prognostic factor for CRC patients. It 
mainly involved in metabolic process and exerted binding and catalytic activities. [b] 


\section{High expression of HSP90 is associated with poor}

3 prognosis in patients with colorectal cancer

4

5

6

7

8

Shuming Zhang ${ }^{1}$, Shichao Guo ${ }^{1}$, Zhangfu $\mathrm{Li}^{1}$, Dan $\mathrm{Li}^{1}$ and Qimin Zhan ${ }^{1,2}$

${ }^{1}$ State Key Laboratory of Molecular Oncology, National Cancer Center/National Clinical

Research Center for Cancer/Cancer Hospital, Chinese Academy of Medical Sciences and Peking Union Medical College. Beijing, China

${ }^{2}$ Laboratory of Molecular Oncology, Key laboratory of Carcinogenesis and Translational Research (Ministry of Education/Beijing), Laboratory of Molecular Oncology, Peking University Cancer Hospital \& Institute, Beijing, China.

Corresponding Authors:

Dan Li and Qimin Zhan are the Corresponding Authors

Dan Li

${ }^{1}$ State Key Laboratory of Molecular Oncology, National Cancer Center/National Clinical Research Center for Cancer/Cancer Hospital, Chinese Academy of Medical Sciences and Peking Union Medical College.17\#Panjiayuan nanli, Chaoyang district, Beijing, China

Email address: eileenld@gmail.com

Qimin Zhan

${ }^{1}$ State Key Laboratory of Molecular Oncology, National Cancer Center/National Clinical Research Center for Cancer/Cancer Hospital, Chinese Academy of Medical Sciences and Peking Union Medical College.17\# Panjiayuan nanli, Chaoyang district, Beijing, China

${ }^{2}$ Laboratory of Molecular Oncology, Key laboratory of Carcinogenesis and Translational Research (Ministry of Education/Beijing), Laboratory of Molecular Oncology, Peking University Cancer Hospital \& Institute, Beijing, China.

Email address: zhanqimin@bjmu.edu.cn

\section{Abstract}

Background: Heat shock protein 90 (HSP90) is a highly conserved chaperone with an approximate molecular weight of $90-\mathrm{kDa}$. It plays a critical role in maintaining stability and homeostasis of oncoproteins, helping cancer cells living in the unsuitable environmental conditions. The current study aims to inquire the difference of HSP90 expression in tumor tissues and normal tissues, analyze the correlation between HSP90 expression and the prognoses of patients with colorectal cancer (CRC), and investigate its role in CRC preliminarily. 
40 Methods: Online analysis of HSP90 mRNA levels in different cancers was firstly done in

41 GEPIA. Then HSP90 expression was determined by immunohistochemistry between 99

42 colorectal cancer (CRC) tissues and 81 normal tissues. Chi-square test or Fisher's exact test was

43 used to analyze the relationship between HSP90 and histopathologic characteristics. Kaplan-

44 Meier analysis and Cox's proportional hazards model were also done for further analysis of the

45 prognostic values of HSP90. Pearson's correlation coefficients between HSP90 expression

46 values and other mRNA expression values were calculated based on TCGA dataset and

47 bioinformatic analysis was done about these screened genes.

48 Results: CRC tissues showed significantly higher expression of HSP90 than normal tissues

49 (55.6\% vs. 3.7\%, P<0.0001). Kaplan-Meier curves showed high HSP90 expression was

50

51

52

53

54

55

56

57

58

59

60

61

62

63

64

65

66

67

68

69

70

71

72

73

74

75

76

77

78

79 associated with poor prognosis $(\mathrm{P}=0.039)$ in $\mathrm{CRC}$ patients, and multivariate Cox proportional hazards regression model analysis also indicated that HSP90 expression ( $\mathrm{HR}=1.930,95 \% \mathrm{CI}$ : 1.113-3.349, $\mathrm{P}=0.019$ ) linked to poor prognosis. Moreover, 85 genes were correlated with HSP90, which were involved in metabolic process and enriched in pathways of Proteasome and Base excision repair.

Conclusions: Our results suggested that HSP90 expression is inversely associated with survival outcomes and could be an independent prognostic factor for CRC patients. It mainly involved in metabolic process and exerted binding and catalytic activities.

\section{Introduction}

Colorectal cancer (CRC) is the third leading malignancy for both genders combined, accounting for approximately 9.2\% cancer-related deaths worldwide. Epidemiologic studies showed an increasing trend in the overall CRC incidence and mortality with an estimated 1849,518 new cases in 2018 (Ferlay, Colombet et al. 2019). Surgical resection is regarded as the standard treatment for locally colorectal cancer, while adjuvant therapy and neoadjuvant therapy are strongly suggested for patients with distant metastasis tumors (Recio-Boiles and Cagir 2019). The prevalence of screening programs for the early detection of CRC patients and novel therapeutic strategies in metastatic patients have greatly improved the general outcomes (Siegel, Miller et al. 2017). However, approximately half of the patients will die within five years after . . diagnosis, especially for patients diagnosed at stage IV, which have a 5-year overall survival rate at around only $10 \%$ (Kuipers, Grady et al. 2015). Therefore, it is important to identify the prognostic biomarkers for the optimized treatments and development of new therapeutic strategies.

HSP90 is one of the most abundant proteins in normal cells, which plays central roles in many signaling and other cellular pathways (Makhnevych and Houry 2012). Previous studies have shown that its expression is significantly higher in tumors than normal cells, helping cancer cells to survive in poor physiological and stress conditions such as hypoxia, nutritional deficiencies, low $\mathrm{pH}$, and exposure to UV light and chemicals (Whitesell and Lindquist 2005). Along with other chaperones, HSP90 participated in the stabilization and maturation of a large number of client proteins, ranging from stress regulation and protein folding to DNA repair, development, 
80

81

82

83

84

85

86

87

88

89

90

91

92

93

94

95

96

97

98

99

100

101

102

103

104

105

106

107

108

109

110

111

112

113

114

115

116

117

118

119

the immune response and many other processes (Echeverria, Bernthaler et al. 2011). Moreover, many of the oncoproteins that contribute to accelerated growth, proliferation and survival of neoplastic cells are client proteins of the HSP90 chaperone complex, including Bcr-Abl, HER-2, EGFR, C-Raf, B-Raf, Akt, Met, VEGFR, FLT3, AR and HIF-1 $\alpha$ (Moran Luengo, Mayer et al. 2019). Thus, HSP90 is an attractive target for drug design and there are already many clinical trials studying the role of HSP90 inhibitors in the process of cancer therapies (Jhaveri, Ochiana et al. 2014).

In this present study, we aimed to demonstrate the correlation between HSP90 and outcomes of colorectal cancer. To achieve this goal, we firstly analyzed the expression of HSP90 protein based on the GEPIA database and the results showed a higher expression of HSP90 in various types of cancers including CRC. In addition, immunohistochemistry analysis of paraffinembedded specimens from 99 patients also proved the up-regulation of HSP90 protein in CRC tissues comparing with adjacent tissue. We further investigated its associations with clinicopathologic factors and prognosis, and the results showed the increased HSP90 expression was related to poor prognosis of CRC patients. Moreover, the main biological pathways that related with HSP90 expression in colorectal cancer were analyzed by using Gene Ontology (GO) and the Kyoto Encyclopedia of Genes and Genomes (KEGG) analysis.

\section{Materials \& Methods}

\section{Patients}

Formalin-fixed paraffin-embedded tumor specimens from surgical resections of the primary tumor were obtained from 99 patients with CRC who underwent curative surgery at our hospital from September 2008 to August 2010. The ethical approval of the study was obtained from the ethics committee of National Cancer Center /Cancer Hospital of Chinese Academy of Medical Sciences and Peking Union Medical College (NCC2018-071). These patients had confirmed pathological diagnosis and complete clinical data, and did not receive radiotherapy or chemotherapy before the surgery. Survival data of all these patients were followed up by telephone interview until October 31, 2018.

\section{Immunohistochemistry (IHC) and evaluation of staining}

Immunohistochemistry assay was performed with $4 \mu \mathrm{m}$ sections of paraffin-embedded tissues on polylysine coated slides. Slides were deparaffinized with xylene and rehydrated through descending gradient ethanol (100\%-95\%-85\%-75\%). Then these slides were washed with phosphate-buffered saline solution (PBS, $0.01 \mathrm{M}, \mathrm{pH} 7.0$ ) for three times. Antigen retrieval was performed by boiling in $10 \mathrm{mM}$ sodium citrate buffer ( $\mathrm{pH}$ 6.0) for 15 mins and then cooled down to room temperature (RT) naturally. Endogenous peroxidase activity was blocked with $3 \%$ hydrogen peroxide at RT for 10 mins. After being washed for three times with PBS, the sections were incubated in 5\% bovine serum albumin at RT for 30 mins to block non-specific binding. Then they were incubated with anti-HSP90 mAb (1:500) (cat: ab59459, Abcam, USA) overnight at $4{ }^{\circ} \mathrm{C}$. After washing with PBS for three times, the specimens were incubated with horseradish peroxidase-conjugated goat anti-rabbit IgG secondary antibodies (1:500) (cat: no.WLA023a, 
120 Wanleibio Co., Ltd.) for $1 \mathrm{~h}$ at RT and visualized by DAB development with Dako EnVison kit

121 (Dako, Glostrup, Denmark). Finally, all slides were counterstained with hematoxylin, dehydrated

122 and mounted with glycerol gelatin. Sections were observed with a microscope.

123 The HSP90 expression-intensity scores were independently determined by two experienced

124 pathologists who were blinded to the information of patients. According to the percentage of the

125 HSP90 positive tumor cells and intensity of staining, the expression area was graded into four

126 levels: $0(0), 1(<1 / 10), 2(1 / 10-1 / 3), 3(1 / 3-2 / 3)$, and $4(\geq 2 / 3)$. The staining intensity score was

127 defined as follows: no staining, 0 ; weak staining, 1; moderate staining, 2; strong staining, 3 . The

128 total HSP90 expression index was generated by multiplying the score of staining intensity and

129 the percentage of HSP90 expression, which ranged from 0 to 12 . We defined scores of $0-3$ as the

130 low expression group, and scores of 4-12 as the high expression group.

131 Analysis of HSP90 genes expression level in different cancers

132 Online analysis of HSP90 mRNA levels of different cancers was done by GEPIA (Gene

133 Expression Profiling Interactive Analysis, http://gepia.cancer-pku.cn/index.html), a web server

134 for cancer and normal gene expression profiling analysis. It worked based on database of the

135 TCGA and the GTEx project including 9736 tumors and 8587 normal samples RNA-Seq data

136 and could be used to analyze gene expression through a standard processing pipeline.

137 Statistical analysis

138 Nonparametric Mann-Whitney U test was used to detect the HSP90 expression difference

139 between cancer tissues and adjacent normal tissues. Chi-square test or Fisher's exact test was

140 performed to analyze the relationship between HSP90 status and CRC patients'

141 clinicopathological features. Survival curves were determined with the Kaplan-Meier method,

142 and different survival rates between groups were compared with the log-rank test. The

143 significance of variables for survival was conducted with the Cox proportional hazards model in

144 univariate and multivariate analysis. All statistical analysis was performed with the SPSS 13.0

145 statistical software (IBM Corp., Armonk, NY, USA). P $<0.05$ (two-tailed) was considered to

146 indicate a statistically significant difference.

147 Bioinformatics analyses

148 We firstly downloaded RNA Seq V2 RSEM data which including 17,989 gene expression of 382

149 CRC tissues from https://www.cbioportal.org/ by using R “cgdsr" package. By calculating

150 Pearson's correlation coefficients, we screened genes which positively correlated with the

151 expression of HSP90 (Pearson's correlation $\geq 0.4, \mathrm{P}<0.0001$ ). Then GO analysis and KEGG

152 analysis were performed using edgeR on OmicShare, an online platform for data analysis

153 (www.omicshare.com/tools). Q value $<0.05$ was used as the thresholds in selecting significant

154 GO and KEGG pathways.

155

156 Results

157 Dataset analysis indicated a significant different expression of HSP90 between cancer

158 tissues and normal tissues

Peer] reviewing PDF | (2019:06:38400:1:2:NEW 6 Sep 2019) 
159 GEPIA is a commodious and intuitive online tool for gene analysis, a web-based tool based on

160

161

162

163

164

165

166

167

168

169

170

171

172

173

174

175

176

177

178

179

180

181

182

183

184

185

186

187

188

189

190

191

192

193

194

195

196

197

198

TCGA and GTEx data. It provides key interactive and customizable functions including differential genes expression analysis, profiling plotting, similar gene detection, correlation analysis, and dimensionality reduction analysis (Tang, Li et al. 2017). We analyzed the HSP90 mRNA expression profile across all tumor samples and normal tissues in GEPIA. From these results showed in bar plot (Fig 1A), we found HSP90 had a different expression between some kinds of cancers and normal tissues. Statistical analysis showed in dot plot indicated significant higher expression in 10 kinds of cancer tissues: breast invasive carcinoma (BRCA), cervical squamous cell carcinoma and endocervical adenocarcinoma (CESC), colon adenocarcinoma (COAD), lymphoid neoplasm diffuse large B-cell lymphoma (DLBC), esophageal carcinoma (ESCA), pancreatic adenocarcinoma (PAAD), rectum adenocarcinoma (READ), skin cutaneous melanoma (SKCM), stomach adenocarcinoma (STAD), and thymoma (THYM) than corresponding normal tissues (Fig 1B). Detailed analyses of the expression of HSP90 of COAD and READ with TCGA data were shown with number of samples in Fig1C.

\section{HSP90 had a higher expression in colon cancer tissues}

Immunohistochemistry was done to evaluate and compare the expression of HSP90 between 99 CRC tissues and 81 adjacent tissues. The expression of HSP90 mainly located in cytoplasm, which had no significant difference between cancer tissues and adjacent tissues (Fig 2). The expressions of HSP90, evaluated by the HSP90 expression score index, were less than 4 points in most normal tissues and were lower than cancer tissues.

In order to make a deeper analysis, we defined score index 0 to 3 as low-level group (including negative expression), 4 to 12 as high-level group. In $99 \mathrm{CRC}$ tissues, 55 (55.6\%) were in the high-level group and the rest $(44.4 \%)$ were in the low-level group. In contrary, 78 out of 81 $(96.3 \%)$ adjacent tissues were in the low-level group. Furthermore, 65 out of $78(80.2 \%)$ adjacent tissues were negative expression. Statistical analysis showed a significantly higher expression of HSP90 in cancer tissues (Nonparametric Mann-Whitney U test, P <0.0001) (Fig 2B).

\section{Association between HSP90 expression and clinicopathological characteristics of CRC patients}

Then we collected clinicopathological parameters of CRC patients and investigated correlations with HSP90 expression (Table 1). As for general clinical characteristics, no significant expression difference was found in sex groups $(\mathrm{P}=0.223)$ or age groups $(\mathrm{P}=0.438)$. Chi-square test and Fisher's exact test were also used to analyze the relationship between HSP90 and some pathological variables which were important for evaluating prognosis. However, no obvious association was found between HSP90 expression levels in CRC tissues and tumor location $(\mathrm{P}=0.604)$, pathological differentiation $(\mathrm{P}=0.621)$, $\mathrm{T}$ phase $(\mathrm{P}=0.505)$, lymph node metastasis $(\mathrm{P}=0.608)$ or AJCC stage $(\mathrm{P}=0.749)$.

\section{High expression of HSP90 predicts poor prognosis of CRC patients}

Survival analysis was conducted to explore whether HSP90 expression influences clinical progression in CRC patients. Here, we did not analyze the relationship between $\mathrm{M}$ category (describes the presence or otherwise of distant metastatic spread) and prognosis as only three

Peer] reviewing PDF | (2019:06:38400:1:2:NEW 6 Sep 2019) 
199

200

201

202

203

204

205

206

207

208

209

210

211

212

213

214

215

216

217

218

219

220

221

222

223

224

225

226

227

228

229

230

231

232

233

234

235

236

237

238

cases had distal metastasis. Besides lymph node metastasis $(\mathrm{P}<0.001)$, AJCC stage $(\mathrm{P}<0.001)$ and pathological differentiation ( $\mathrm{P}=0.033)$, high HSP90 expression was associated with poor prognosis $(\mathrm{P}=0.039)$ in CRC patients as well. HSP90 high-expression group have a significantly worse overall mean of survival (OS) than low-expression group (51.6 vs. 66.0 months) (Fig 3).

Next, Cox's proportional hazards model was also done for further analysis of prognostic value of HSP90 expression and other clinical parameters (Table 2). These results revealed that variables including lymph node metastasis $(\mathrm{HR}=2.192,95 \% \mathrm{CI}: 1.544-3.112, \mathrm{P}<0.001)$, pathological differentiation ( $\mathrm{HR}=1.737,95 \% \mathrm{CI}: 1.035-2.915, \mathrm{P}=0.037)$ and high HSP90 expression ( $\mathrm{HR}=1.738,95 \% \mathrm{CI}: 1.018-2.966, \mathrm{P}=0.043)$ linked to poor prognosis (Fig 4A). Then multivariate analysis of Cox's proportional hazards regression model was further done to exclude the false positive results caused by the interaction of different factors. Multivariate analysis results revealed that, in addition to the acknowledgeable factors including lymph node metastasis $(\mathrm{HR}=2.459,95 \% \mathrm{CI}: 1.652-3.661, \mathrm{P}<0.001)$ and pathological differentiation $(\mathrm{HR}=1.749,95 \%$ CI: 1.017-3.007, $\mathrm{P}=0.043)$, HSP90 expression level ( $\mathrm{HR}=1.930,95 \% \mathrm{CI}: 1.113-3.349, \mathrm{P}=0.019)$ could be an independent prognostic factor for CRC patient prognosis prediction (Fig 4B).

\section{HSP90-related signaling pathways based on TCGA database}

We performed functional enrichment analysis to explore the biological pathway and process correlated with HSP90. Firstly, the Pearson's correlation coefficients between HSP90 expression values and other mRNA expression values were calculated in the TCGA dataset. 85 genes were positively correlated with the expression of HSP90 (defined as Pearson's correlation coefficient $\geqslant 0.4$, as shown in Supplementary Data 1) and included in the GO analysis (Fig 5) and the KEGG analysis, respectively (as shown in supplementary data). The GO analysis results showed that it mainly existed as an intracellular molecular (Fig 5D), which were involved in metabolic process (Fig 5B) and exerted binding and catalytic activity (Fig 5C). Besides, KEGG analysis enriched these genes in pathways of Proteasome and Base excision repair (Fig 6).

\section{Discussion}

Colorectal cancer was one of the most frequent tumor types worldwide and approximately half of the patients will die within 5 years after diagnosis (Siegel, Miller et al. 2018). Therefore, it is of great importance to identify novel biomarkers and therapeutic targets, for the improvement of clinical outcomes for CRC patients. Hsp90 is a highly conserved and ubiquitously expressed molecular chaperone that involved in the folding, maturation, and degradation of client proteins (Sima and Richter 2018). It participates in essential cellular activities by supporting the maturation process of its client proteins, most of which are involved in cell growth, proliferation and survival, which are critical functions for neoplastic cells (Kryeziu, Bruun et al. 2019).

In this study, the HSP90 mRNA expression across all tumor samples and normal tissues based on GEPIA dataset were analyzed. The results indicated significant elevated expressions in multiple cancer types including CRC, consistent with the previous studies that reported elevated HSP90 expressions in breast cancer, lung cancer, melanoma and myeloid leukemia (Yano, Naito et al. 1999, Becker, Multhoff et al. 2004, Biaoxue, Xiling et al. 2012, Gonzalez, De Brasi et al. 
239

240

241

242

243

244

245

246

247

248

249

250

251

252

253

254

255

256

257

258

259

260

261

262

263

264

265

266

267

268

269

270

271

272

273

274

275

276

277

278

2014). Additionally, a higher protein expression level of HSP90 was further observed in 99 CRC tissues compared with 81 adjacent normal tissues by IHC assay. The increased HSP90 expression was significantly associated with poor prognosis of CRC patients, revealed by survival curve analysis. Our results indicated both mRNA and protein levels of HSP90 were elevated in tumor tissues, which suggested that HSP90 gene had a higher activity in both transcriptional and translational levels in CRC tissues.

To further investigate the association between the clinical characteristics and prognosis, Cox's regression analysis was performed. The results suggested that tumor lymph node metastasis, differentiation status, AJCC stage (American Joint Committee on Cancer, an organization best known for defining and popularizing cancer staging standards according, officially the AJCC staging system) and HSP90 expression were the independent risk factors impacting the OS of CRC patients. Therefore, we speculated that HSP90 might act as an oncogene and could be considered as a potential therapeutic target for the clinical management of CRC. However, more mechanistic studies are required to further support this conclusion. Besides, as a secretable protein (Tsutsumi and Neckers 2007), the serum HSP90 abundance of CRC patients could also be measured to evaluate the application in liquid biopsy.

Owing to the importance of HSP90 in the regulation of different cellular proteins, it has become an attractive therapeutic target for many cancers (Schopf, Biebl et al. 2017). Currently, a number of specific HSP90 inhibitors have been evaluated in preclinical or clinical trials (Kryeziu, Bruun et al. 2019). The first-generation inhibitors are mainly natural products, including Geldanamycin (GA) and radicicol (RD), which competitively bind with the N-terminal of ATP-binding pocket and prevent the ATP or ADP binding of HSP90 (Roe, Prodromou et al. 1999). However, clinical applications of the first-generation inhibitors were limited due to physicochemical properties and hepatotoxicity (Neckers and Workman 2012). Therefore, more attentions were paid on the derivatives of GA and RD, so-called the second-generation inhibitors, including 17-AAG (tanespimycin), 17-DMAG (alvespimycin), IPI-504 (retaspimycin hydrochloride), KF25706 and KF58333 (Miyata 2005, Porter, Ge et al. 2009). In spite of better solubility and lower toxicological properties, none of these inhibitors have been approved as a new drug due to the unsatisfactory antitumor effects (Soga, Akinaga et al. 2013).

General reasons for the unsatisfactory therapeutic effects of HSP90 inhibitors have been described. One possible explanation is that the overexpression of some related proteins, such as Heat shock factor protein 1 (HSF1), P-glycoprotein 1 (P-gp) and UDP glucuronosyltransferase 1A (UGT1A), may be associated with resistance of HSP90 inhibitors (Kryeziu, Bruun et al. 2019). However, the mechanistic aspects of HSP90 are still need to be investigated for improving the efficacy of HSP90 inhibitors.

Hence, the current study screened out 85 genes which were positively correlated with the expression of HSP90 and performed functional enrichment analysis to explore the biological pathways and processes correlated with HSP90. According to the GO analysis, the 85 genes mainly enriched in three biological processes embedding metabolic process, single organism process and response to stimuli. Additionally, these genes showed significant enrichment in 
279

280

281

282

283

284

285

286

287

288

289

290

291

292

293

294

295

296

297

298

299

300

301

302

303

304

305

306

307

308

309

310

311

312

313

314

315

316

317

318

319

320

molecular function of binding and catalytic activity. Besides, KEGG analysis enriched these genes in pathways of proteasome and base excision repair.

Consistent with our results, HSP90 is a "stress sensor" that facilitating in several cellular functions through regulating of folding and assembly of its client proteins. In recent years, it has also been reported that HSP90 chaperones participated in oncogene-driven metabolic rewiring, which is one of the hallmarks of cancer cells (Condelli, Crispo et al. 2019). Obviously, HSP90 is closely cooperate with ubiquitin-proteasome machinery to control protein homeostasis (Makhnevych and Houry 2012). Moreover, DMAG, a HSP90 inhibitor, was reported to interfere with base excision repair and ATM-mediated DNA repair (Koll, Feis et al. 2008). Taken together, the functional enrichment analyses of these HSP90 related genes may help us have a better understanding of HSP90 functions and implicate the application of rational drug combinations.

\section{Conclusions}

Collectively, we found HSP90 expression was upregulated in CRC tissues compared with normal tissues, and its high expression was associated with poor survival in CRC patients. Additionally, HSP90 expression was the independent risk factor that influencing the OS of CRC patients. Furthermore, bioinformatics analyses showed that the co-expression genes of HSP90 mainly involved in metabolic process, proteasome and base excision repair. These results suggested that high HSP90 expression may be used as an indicator of poor prognosis and predicted its roles in therapy for CRC patients.

\section{Acknowledgements}

We thank Dr. Lin Feng for Bioinformatic analysis of HSP90 related genes.

\section{References}

Biaoxue, R., J. Xiling, Y. Shuanying, Z. Wei, C. Xiguang, W. Jinsui and Z. Min (2012). "Upregulation of Hsp90-beta and annexin A1 correlates with poor survival and lymphatic metastasis in lung cancer patients." J Exp Clin Cancer Res 31: 70.

Condelli, V., F. Crispo, M. Pietrafesa, G. Lettini, D. S. Matassa, F. Esposito, M. Landriscina and F. Maddalena (2019). "HSP90 Molecular Chaperones, Metabolic Rewiring, and Epigenetics: Impact on Tumor Progression and Perspective for Anticancer Therapy." Cells 8(6).

Echeverria, P. C., A. Bernthaler, P. Dupuis, B. Mayer and D. Picard (2011). "An interaction network predicted from public data as a discovery tool: application to the Hsp90 molecular chaperone machine." PLoS One 6(10): e26044.

Ferlay, J., M. Colombet, I. Soerjomataram, C. Mathers, D. M. Parkin, M. Pineros, A. Znaor and F. Bray (2019). "Estimating the global cancer incidence and mortality in 2018: GLOBOCAN sources and methods." Int J Cancer 144(8): 1941-1953.

Gonzalez, M., C. De Brasi, C. Ferri, R. Bengio, M. Bianchini and I. Larripa (2014). "CAMKIIgamma, HSP70 and HSP90 transcripts are differentially expressed in chronic myeloid leukemia cells from patients with resistant mutated disease." Leuk Lymphoma 55(9): 2101-2108. Jhaveri, K., S. O. Ochiana, M. P. Dunphy, J. F. Gerecitano, A. D. Corben, R. I. Peter, Y. Y. 
321

322

323

324

325

326

327

328

329

330

331

332

333

334

335

336

337

338

339

340

341

342

343

344

345

346

347

348

349

350

351

352

353

354

355

356

357

358

359

360

361

362

363

364

365

366

Janjigian, E. M. Gomes-DaGama, J. Koren, 3rd, S. Modi and G. Chiosis (2014). "Heat shock protein 90 inhibitors in the treatment of cancer: current status and future directions." Expert Opin Investig Drugs 23(5): 611-628.

Koll, T. T., S. S. Feis, M. H. Wright, M. M. Teniola, M. M. Richardson, A. I. Robles, J. Bradsher, J. Capala and L. Varticovski (2008). "HSP90 inhibitor, DMAG, synergizes with radiation of lung cancer cells by interfering with base excision and ATM-mediated DNA repair." Mol Cancer Ther 7(7): 1985-1992.

Kryeziu, K., J. Bruun, T. K. Guren, A. Sveen and R. A. Lothe (2019). "Combination therapies with HSP90 inhibitors against colorectal cancer." Biochim Biophys Acta Rev Cancer 1871(2): 240-247.

Kuipers, E. J., W. M. Grady, D. Lieberman, T. Seufferlein, J. J. Sung, P. G. Boelens, C. J. van de Velde and T. Watanabe (2015). "Colorectal cancer." Nat Rev Dis Primers 1: 15065.

Makhnevych, T. and W. A. Houry (2012). "The role of Hsp90 in protein complex assembly." Biochim Biophys Acta 1823(3): 674-682.

Miyata, Y. (2005). "Hsp90 inhibitor geldanamycin and its derivatives as novel cancer chemotherapeutic agents." Curr Pharm Des 11(9): 1131-1138.

Moran Luengo, T., M. P. Mayer and S. G. D. Rudiger (2019). "The Hsp70-Hsp90 Chaperone Cascade in Protein Folding." Trends Cell Biol 29(2): 164-177.

Neckers, L. and P. Workman (2012). "Hsp90 molecular chaperone inhibitors: are we there yet?" Clin Cancer Res 18(1): 64-76.

Porter, J. R., J. Ge, J. Lee, E. Normant and K. West (2009). "Ansamycin inhibitors of Hsp90: nature's prototype for anti-chaperone therapy." Curr Top Med Chem 9(15): 1386-1418.

Recio-Boiles, A. and B. Cagir (2019). Cancer, Colon. StatPearls. Treasure Island (FL).

Roe, S. M., C. Prodromou, R. O'Brien, J. E. Ladbury, P. W. Piper and L. H. Pearl (1999).

"Structural basis for inhibition of the Hsp90 molecular chaperone by the antitumor antibiotics radicicol and geldanamycin." J Med Chem 42(2): 260-266.

Schopf, F. H., M. M. Biebl and J. Buchner (2017). "The HSP90 chaperone machinery." Nat Rev Mol Cell Biol 18(6): 345-360.

Siegel, R. L., K. D. Miller, S. A. Fedewa, D. J. Ahnen, R. G. S. Meester, A. Barzi and A. Jemal (2017). "Colorectal cancer statistics, 2017." CA Cancer J Clin 67(3): 177-193.

Siegel, R. L., K. D. Miller and A. Jemal (2018). "Cancer statistics, 2018." CA Cancer J Clin 68(1): 7-30.

Sima, S. and K. Richter (2018). "Regulation of the Hsp90 system." Biochim Biophys Acta Mol Cell Res 1865(6): 889-897.

Soga, S., S. Akinaga and Y. Shiotsu (2013). "Hsp90 inhibitors as anti-cancer agents, from basic discoveries to clinical development." Curr Pharm Des 19(3): 366-376.

Tang, Z., C. Li, B. Kang, G. Gao, C. Li and Z. Zhang (2017). "GEPIA: a web server for cancer and normal gene expression profiling and interactive analyses." Nucleic Acids Res 45(W1): W98-W102.

Tsutsumi, S. and L. Neckers (2007). "Extracellular heat shock protein 90: a role for a molecular chaperone in cell motility and cancer metastasis." Cancer Sci 98(10): 1536-1539.

Whitesell, L. and S. L. Lindquist (2005). "HSP90 and the chaperoning of cancer." Nat Rev Cancer 5(10): 761-772.

Yano, M., Z. Naito, M. Yokoyama, Y. Shiraki, T. Ishiwata, M. Inokuchi and G. Asano (1999). "Expression of hsp90 and cyclin D1 in human breast cancer." Cancer Lett 137(1): 45-51.

Peer] reviewing PDF | (2019:06:38400:1:2:NEW 6 Sep 2019) 
367

368

369

370

371

372

373

374

375

376

377

378

379

380

381

382

383

384

385

386

387

388

389

390

391

392

393

394

395

396

397

398

399

400

401

402

403

\section{Figure legends}

\section{Figure 1: Analysis of HSP90 genes expression levels from online databse.}

HSP90 gene expression profile (mRNA level) across all tumor samples and normal tissues were analyzed in GEPIA and these results were shown in bar plot (A) and dot plot (B) separately. Expression levels of HSP90 gene in rectal cancers (C), colon cancers (D) and normal tissues were analyzed using TCGA data. The abbreviations: Adrenocortical carcinoma (ACC), bladder urothelial carcinoma (BLCA), breast invasive carcinoma (BRCA), cervical squamous cell carcinoma and endocervical adenocarcinoma (CESC), cholangio carcinoma (CHOL), colon adenocarcinoma (COAD), lymphoid neoplasm diffuse large B-cell lymphoma (DLBC), esophageal carcinoma (ESCA), glioblastoma multiforme (GBM), head and neck squamous cell carcinoma (HNSC), kidney chromophobe (KICH), kidney renal clear cell carcinoma (KIRC), kidney renal papillary cell carcinoma (KIRP), acute myeloid leukemia (LAML), brain lower grade glioma (LGG), liver hepatocellular carcinoma (LIHC), lung adenocarcinoma (LUAD), lung squamous cell carcinoma (LUSC), ovarian serous cystadenocarcinoma (OV), pancreatic adenocarcinoma (PAAD), pheochromocytoma and paraganglioma (PCPG), prostate adenocarcinoma (PRAD), sarcoma (SARC), skin cutaneous melanoma (SKCM), stomach adenocarcinoma (STAD), testicular germ cell tumors (TGCT), thyroid carcinoma (THCA), thymoma (THYM), uterine corpus endometrial carcinoma (UCEC), uterine carcinosarcoma (UCS) and rectum adenocarcinoma (READ).

\section{Figure 2: Immunohistochemistry and statistical results of HSP90 expression.}

(A-H) The expression of HSP90 in colonic cancer and matched adjacent normal epithelial tissues was detected by immunohistochemistry. Original magnification: $40 \mathrm{x}$ and $200 \mathrm{x}$. (I) Statistical analysis was done by comparing the final scores in colon cancer and adjacent normal epithelial tissues. Colon cancer tissues had a significant higher expression than matched adjacent normal epithelial tissues.

\section{Figure 3: Kaplan-Meier survival analysis in subsets of CRC patients.}

(A) Kaplan-Meier survival analysis of CRC patients according to lymph node metastasis. (B) Kaplan-Meier survival analysis of CRC patients according to AJCC stage. (C) Kaplan-Meier survival analysis of CRC patients according to pathological differentiation grade. (D) KaplanMeier survival analysis of CRC patients according to HSP90 expression. 
404 Figure 4: Forest graphs of Cox proportional hazards model analysis of variables affecting 405 overall survival in colorectal cancer patients.

406 A was univariate analysis results and B was multivariate analysis results.

407

408 Figure 5: Enriched GO analysis of identified genes.

409 (A) The results are summarized in three main categories: biological processes, molecular

410 functions and cellular components. (B) The top 20 enriched GO biological processes terms. (C)

411 The top 20 enriched GO molecular functions terms. (D) The top 20 enriched GO cellular

412 components terms.

413

414 Figure 6: KEGG pathway classification of identified genes.

415 (A) Distribution of the KEGG pathways of identified genes is shown as a bar chart. The

416 horizontal axis is the number of genes, whereas the vertical ordinates are the terms of the KEGG

417 pathways. $\mathrm{Q}<0.05$ was used as the thresholds in selecting significant KEGG pathways. (B) The

418 significant pathways of KEGG enrichment.

419 


\section{Figure 1}

Analysis of HSP90 genes expression level s from online databse .

HSP90 gene expression profile (mRNA level) across all tumor samples and normal tissues were analyzed in GEPIA and these results were shown in bar plot (A) and dot plot (B) separately. Expression levels of HSP90 gene in rectal cancers $(C)$, colon cancers $(D)$ and normal tissues were analyzed using TCGA data. The abbreviations: Adrenocortical carcinoma (ACC), bladder urothelial carcinoma (BLCA), breast invasive carcinoma (BRCA), cervical squamous cell carcinoma and endocervical adenocarcinoma (CESC), cholangio carcinoma ( $\mathrm{CHOL})$, colon adenocarcinoma (COAD), lymphoid neoplasm diffuse large B-cell lymphoma (DLBC), esophageal carcinoma (ESCA), glioblastoma multiforme (GBM), head and neck squamous cell carcinoma (HNSC), kidney chromophobe (KICH), kidney renal clear cell carcinoma (KIRC), kidney renal papillary cell carcinoma (KIRP), acute myeloid leukemia (LAML), brain lower grade glioma (LGG), liver hepatocellular carcinoma (LIHC), lung adenocarcinoma (LUAD), lung squamous cell carcinoma (LUSC), ovarian serous cystadenocarcinoma (OV), pancreatic adenocarcinoma (PAAD), pheochromocytoma and paraganglioma (PCPG), prostate adenocarcinoma (PRAD), sarcoma (SARC), skin cutaneous melanoma (SKCM), stomach adenocarcinoma (STAD), testicular germ cell tumors (TGCT), thyroid carcinoma (THCA), thymoma (THYM), uterine corpus endometrial carcinoma (UCEC), uterine carcinosarcoma (UCS) and rectum adenocarcinoma (READ). 

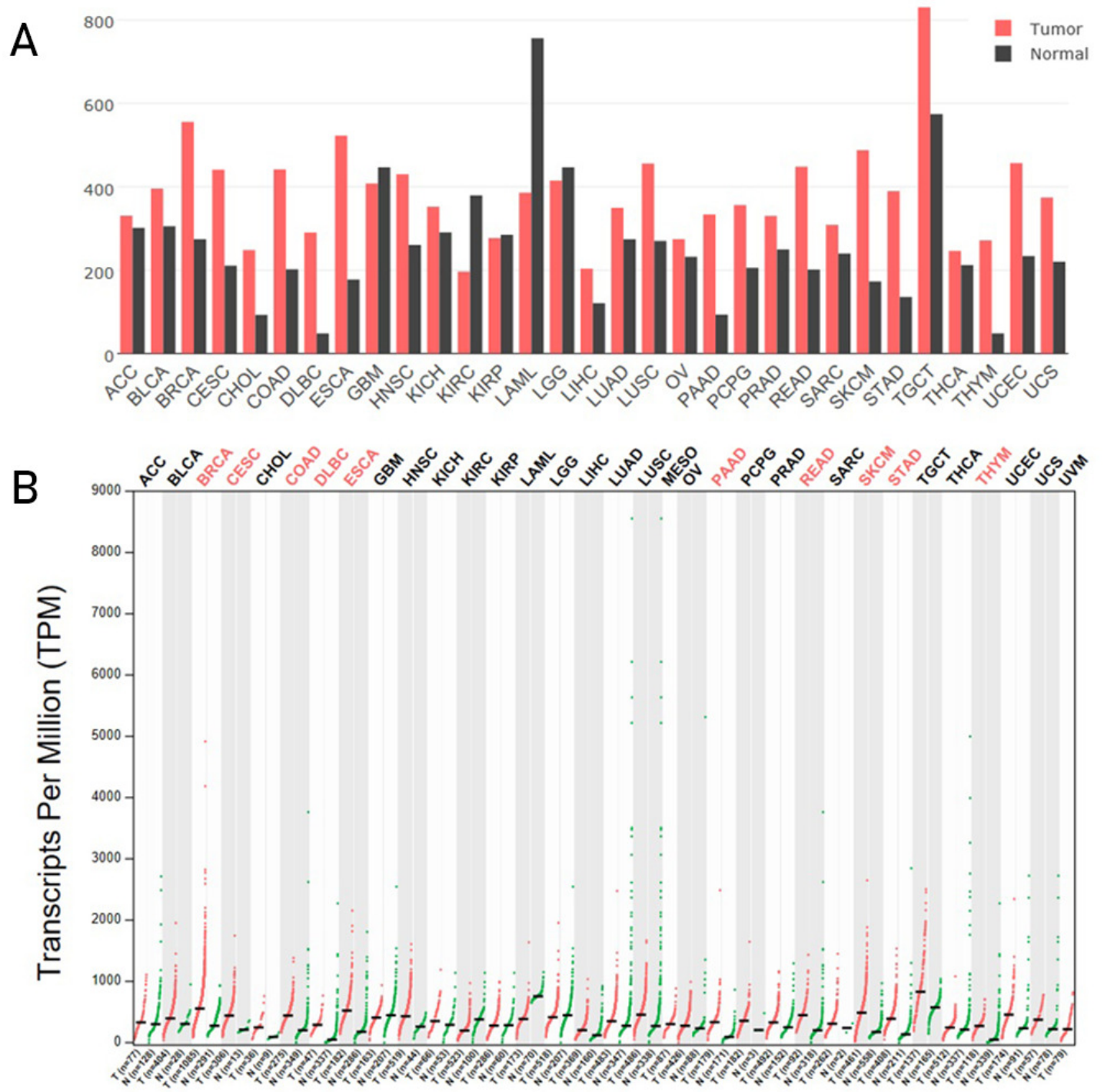

C

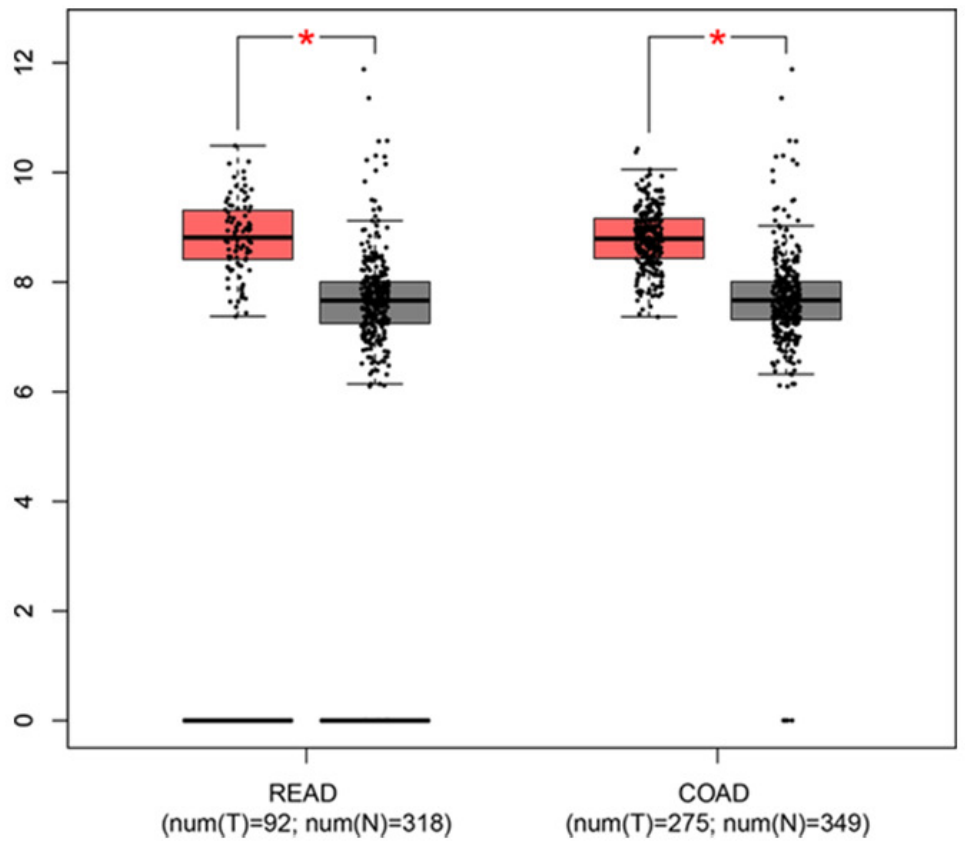




\section{Figure 2}

Immunohistochemistry and statistical results of HSP90expression.

(A -H ) The expression of HSP90 in colonic cancer and matched adjacent normal epithelial tissues was detected by immunohistochemistry. Original magnification: $40 \mathrm{x}$ and $200 \mathrm{x}$. ( I ) Statistical analysis was done by comparing the final scores in colon cancer and adjacent normal epithelial tissues. Colon cancer tissues had a significant higher expression than matched adjacent normal epithelial tissues. 

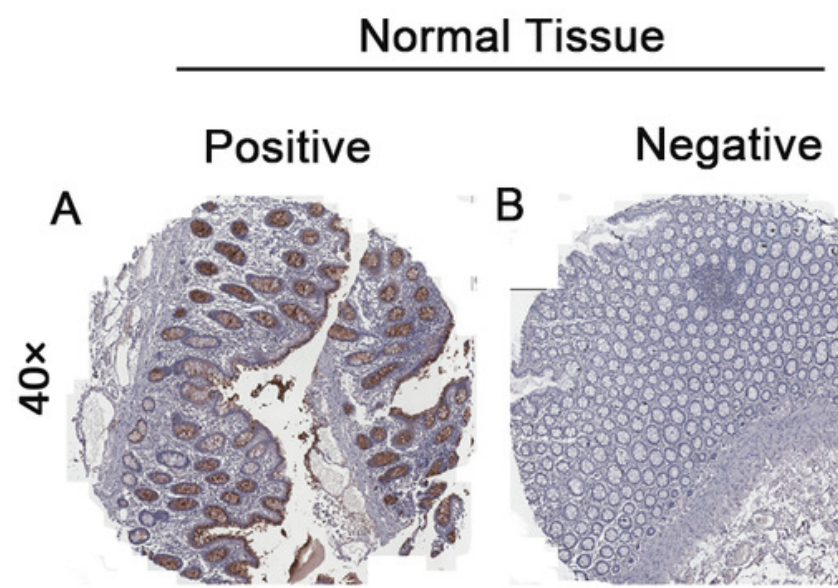

B
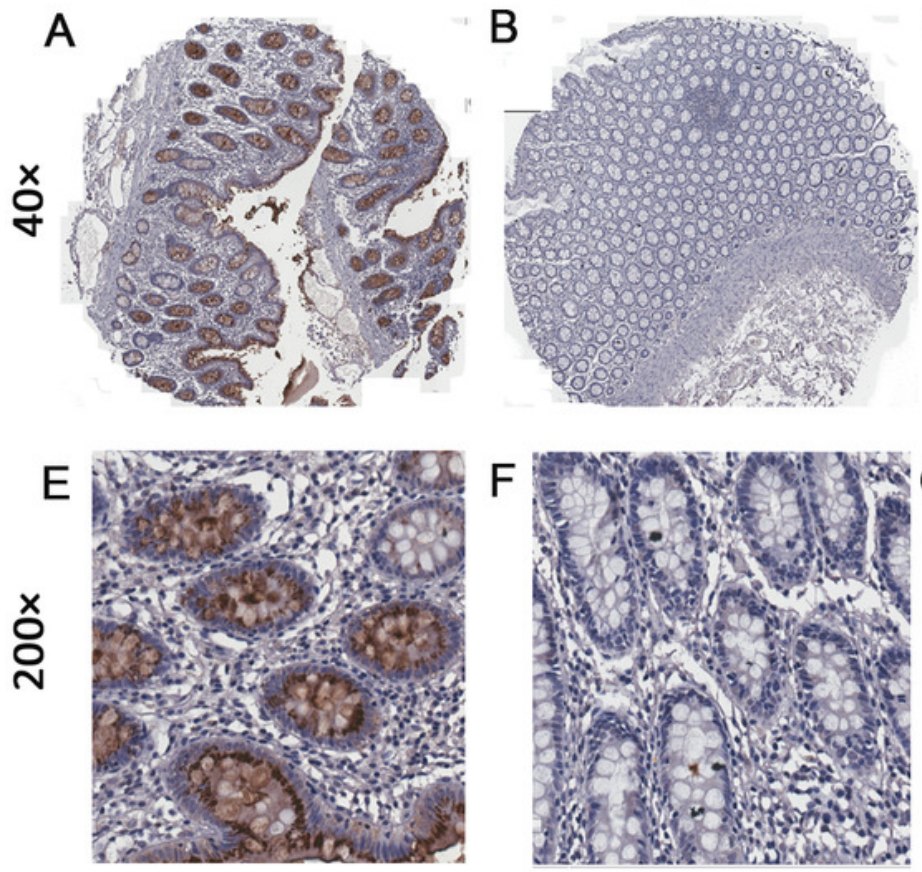

F

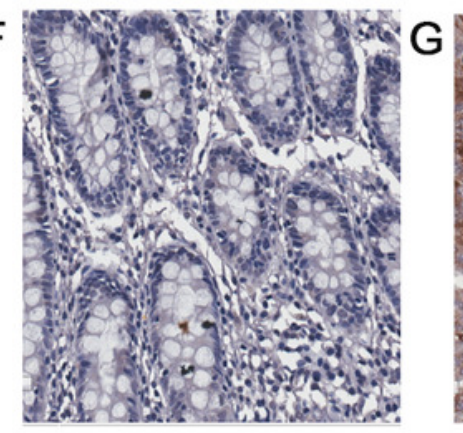

C

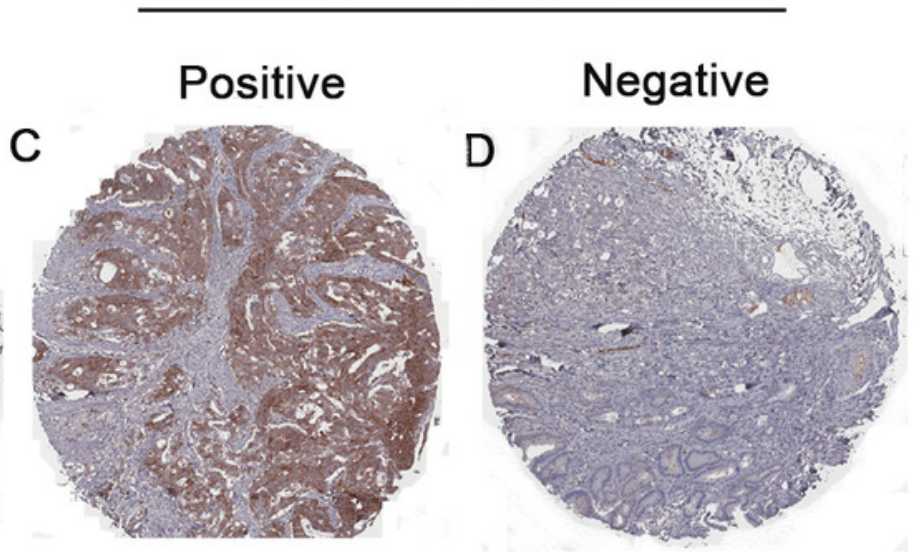

Tumor Tissue
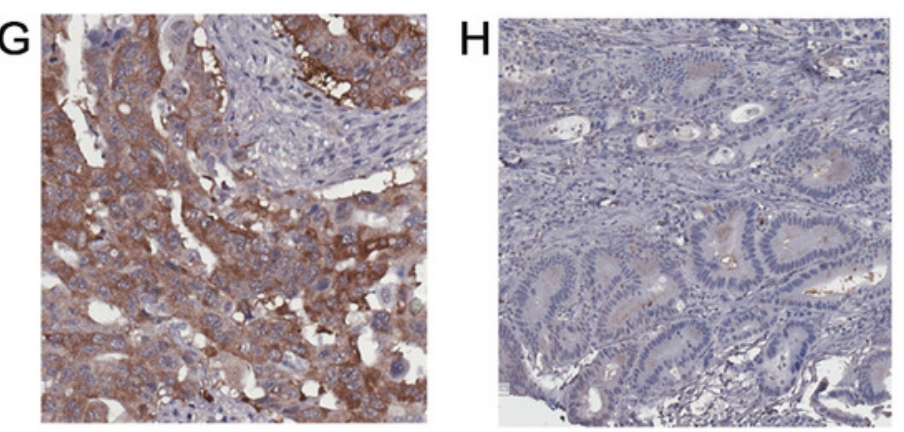

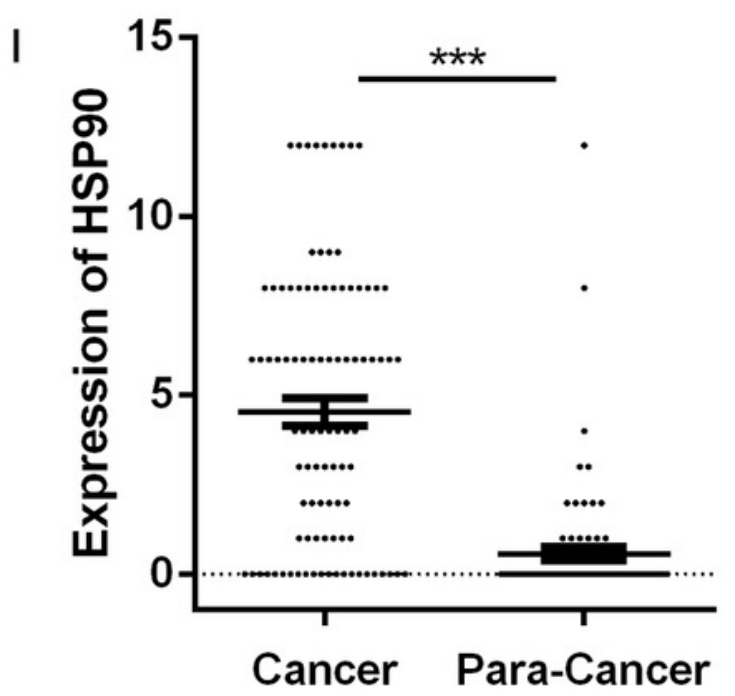




\title{
Figure 3
}

\section{Kaplan-Meier survival analysis in subsets of CRCpatients .}

\begin{abstract}
(A) Kaplan-Meier survival analysis of CRC patients according to lymph node metastasis . (B) Kaplan-Meier survival analysis of CRC patients according to AJCC stage. (C) K aplan-Meier survival analysis of CRC patients according to pathological differentiation grade. (D) Kaplan-Meier survival analysis of CRC patients according to HSP90 expression.
\end{abstract}



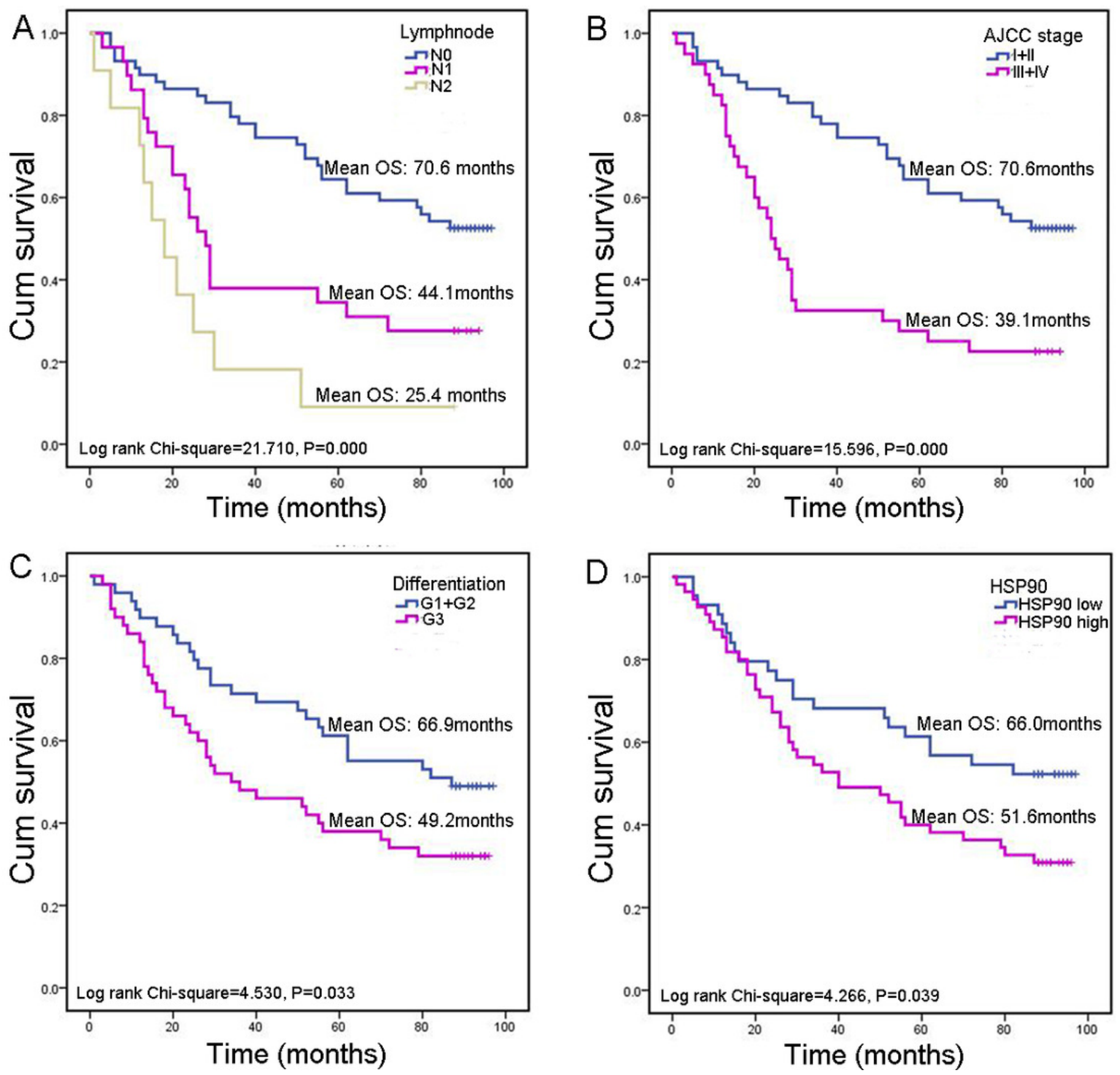
Figure 4

Forestgraphs of Cox proportional hazards model analysis of variables affectingoverall survival in colorectal cancer patients.

A was univariate analysis results and B was multivariate analysis results.
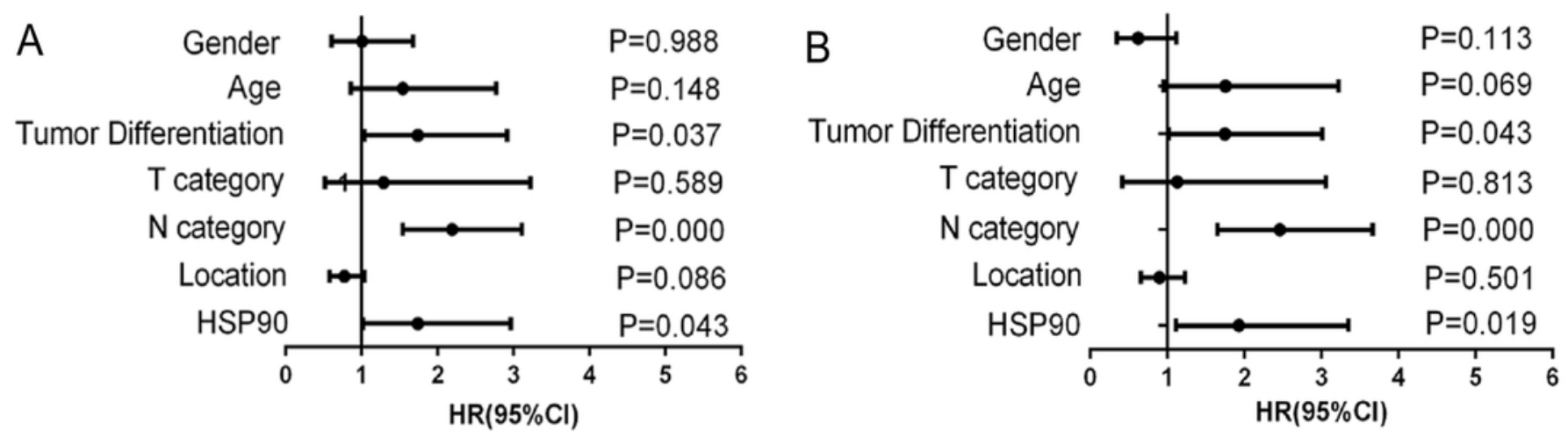
Figure 5

Enriched GO analysis of identified genes.

(A) The results are summarized in three main categories: biological processes, molecular functions and cellular components . (B) The top 20 enriched GO biological processes terms. (C) The top 20 enriched GO molecular functions terms. (D) The top 20 enriched GO cellular components terms.
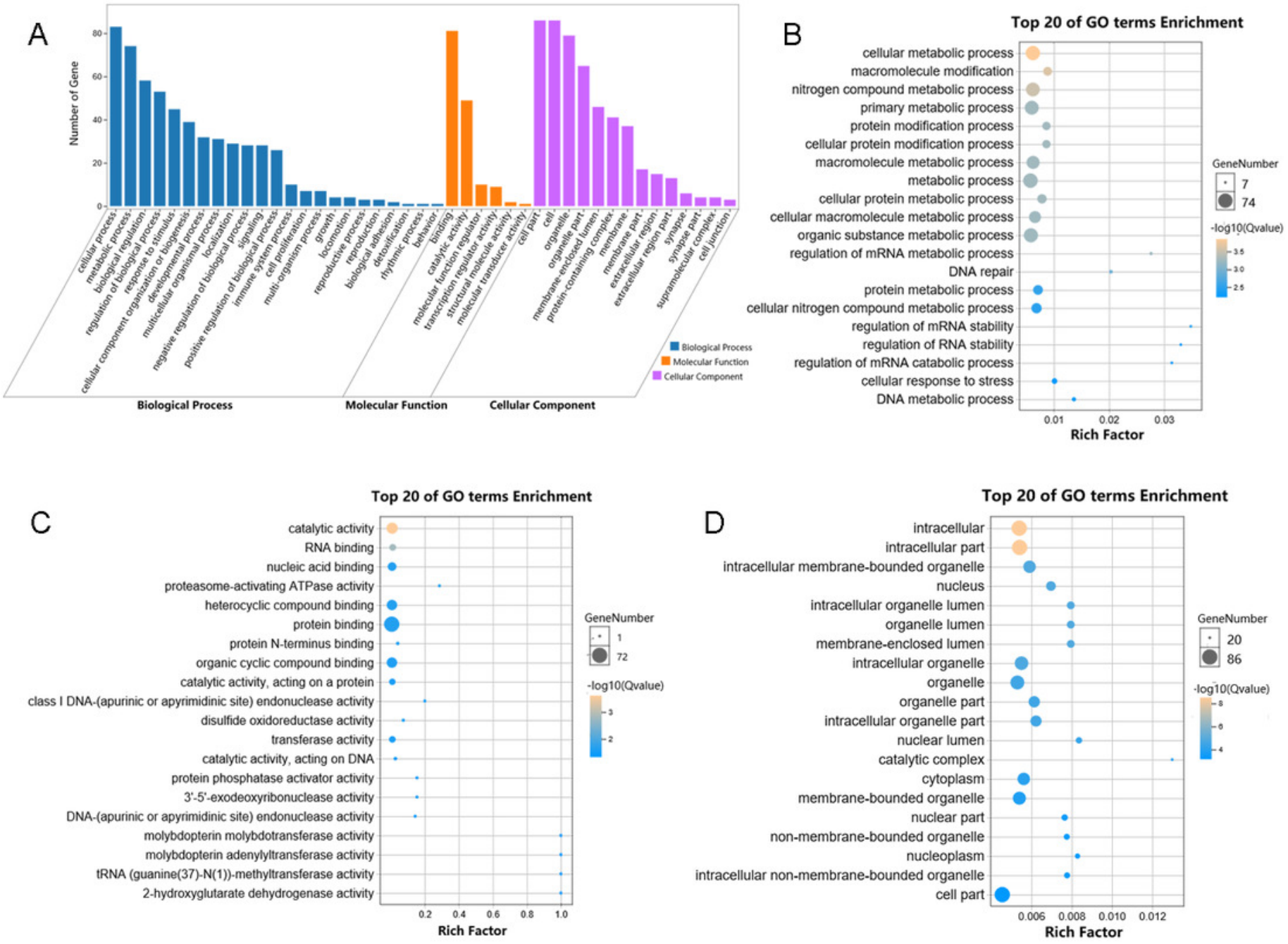
Figure 6

KEGG pathwayclassification of identified genes.

(A) Distribution of the KEGG pathways of identified genes is shown as a bar chart. The horizontal axis is the number of genes, whereas the vertical ordinates are the terms of the KEGG pathways. Q<0.05 was used as the thresholds in selecting significant KEGG pathways. (B) The significant pathways of KEGG enrichment.

A

KEGG pathway annotation

Genetic Information Processing Folding, sorting and degradation Replication and repair -

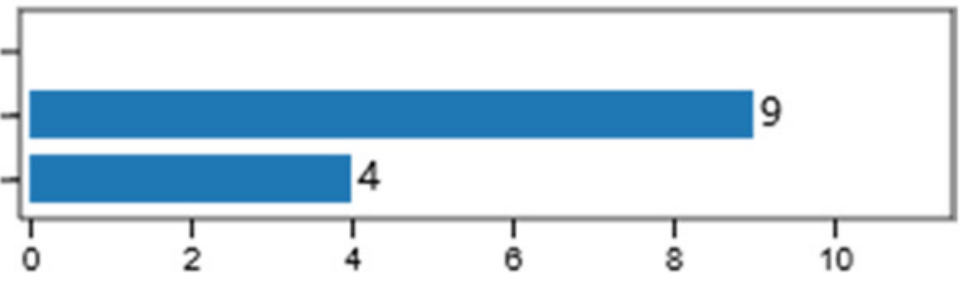

Number of Gene

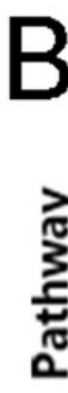

\section{Top 20 of Pathway Enrichment}

究

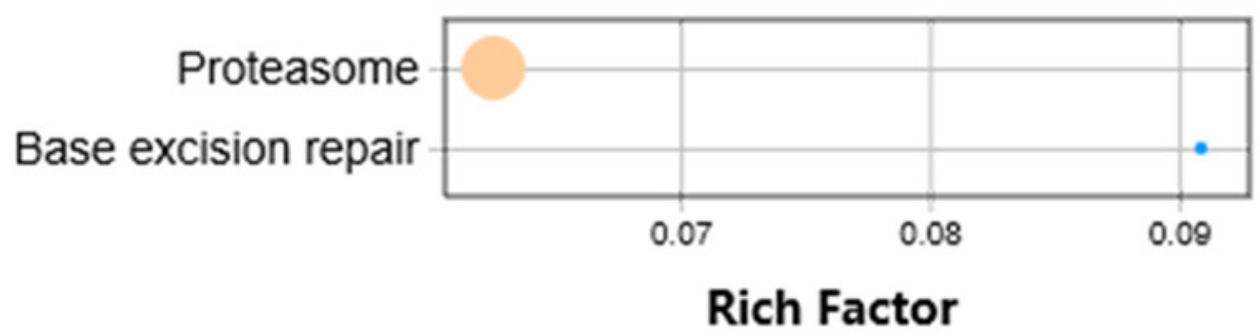

GeneNumber

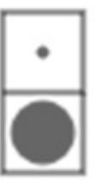

3

4

-log10(Qvalue) 


\section{Table $\mathbf{1}$ (on next page)}

Association between HSP90 expression andclinicopathological characteristics of patients. 
1 Table 1. Association between HSP90 expression and clinicopathological characteristics of patients.

\begin{tabular}{lrrr}
\hline Characteristics & Total cases (\%) & \multicolumn{2}{c}{ HSP90 expression } \\
\cline { 3 - 4 } & & Low (\%) & High (\%) \\
\hline Gender & & & \\
Male & $54(54.5 \%)$ & $27(50.0 \%)$ & $27(50.0 \%)$ \\
Female & $45(45.5 \%)$ & $17(37.8 \%)$ & $28(62.2 \%)$ \\
Age (Year) & & & \\
$<65$ & $31(31.3 \%)$ & $12(38.7 \%)$ & $19(61.3 \%)$ \\
$\geq 65$ & $68(68.7 \%)$ & $32(47.1 \%)$ & $36(52.9 \%)$
\end{tabular}

\section{Location}

Ascending colon

$$
35(35.4 \%) \quad 17(48.6 \%) \quad 18(51.4 \%)
$$

Transverse colon $25(25.3 \%) \quad 9(36.0 \%) \quad 16(64.0 \%)$

Descending-Sigmoid $39(39.4 \%)$

$18(46.2 \%) \quad 21(53.8 \%)$ colon

T category

$\mathrm{T} 1+\mathrm{T} 2$

$$
10(10.1 \%) \quad 3(30.0 \%) \quad 7(70.0 \%)
$$

$\mathrm{T} 3+\mathrm{T} 4$

$89(89.9 \%)$

$41(46.1 \%) \quad 48(53.9 \%)$

$\mathrm{N}$ category

$59(59.6 \%) \quad 27(45.8 \%) \quad 32(54.2 \%)$

N0

$29(29.3 \%) \quad 11(37.9 \%) \quad 18(62.1 \%)$

N1

$11(11.1 \%)$

$6(54.5 \%)$

$5(45.5 \%)$

\section{Differentiation}

Well to moderate

$$
\begin{array}{lll}
49(49.5 \%) & 23(46.9 \%) & 26(53.1 \%) \\
50(50.5 \%) & 21(42.0 \%) & 29(58.0 \%)
\end{array}
$$

Low

\section{AJCC stage}

I, II

$59(59.6 \%)$

$27(45.8 \%)$

$32(54.2 \%)$

III, IV

$40(40.4 \%)$

$17(42.5 \%)$

$23(57.5 \%)$

\section{Overall survival}

Death

$$
59(59.6 \%) \quad 21(35.6 \%) \quad 38(64.4 \%)
$$

0.608

0.621

$$
0.749
$$

0.039 
Alive

$40(40.4 \%)$

$23(57.5 \%)$

$17(42.5 \%)$

2

Peer] reviewing PDF | (2019:06:38400:1:2:NEW 6 Sep 2019) 


\section{Table 2 (on next page)}

Cox proportional hazards model analysis ofvariables affecting overall survival in colorectal cancer patients. 
1 Table 2. Cox proportional hazards model analysis of variables affecting overall survival in colorectal cancer 2 patients

\begin{tabular}{cccccc}
\hline Variable & Categories & \multicolumn{2}{c}{ Univariate analysis } & \multicolumn{2}{c}{ Multivariate analysis } \\
\cline { 3 - 6 } & & $\mathrm{HR}(95 \% \mathrm{CI})$ & $\mathrm{P}$ & $\mathrm{HR}(95 \% \mathrm{CI})$ & $\mathrm{P}$ \\
\hline Gender & Male vs. Female & $1.004(0.600-1.679)$ & 0.988 & $0.620(0.344-1.119)$ & 0.113 \\
Age(years) & $<65$ vs. $\geq 65$ & $1.542(0.858-2.773)$ & 0.148 & $1.756(0.957-3.221)$ & 0.069 \\
Differentiation & Poor vs. Well +Moderate & $1.737(1.035-2.915)$ & 0.037 & $1.749(1.017-3.007)$ & 0.043 \\
T category & T1+T2 vs. T3+T4 & $1.288(0.515-3.221)$ & 0.589 & $1.128(0.416-3.058)$ & 0.813 \\
N category & N0 vs. N1 vs. N2 & $2.192(1.544-3.112)$ & 0.000 & $2.459(1.652-3.661)$ & 0.000 \\
Location & Ascending vs. Transverse vs. & $0.773(0.576-1.037)$ & 0.086 & $0.899(0.658-1.227)$ & 0.501 \\
& Descending-Sigmoid & & & & \\
HSP90 & High vs. Low & $1.738(1.018-2.966)$ & 0.043 & $1.930(1.113-3.349)$ & 0.019 \\
\hline
\end{tabular}

3 Note: HR, hazard ratio; 95\% CI, 95\% confidence interval 